Journal of Mathematics and Statistics 7 (2): 116-123, 2011

ISSN 1549-3644

(C) 2010 Science Publications

\title{
Discretized Scheme Examining Convergence and Geometric Ratio Profiles for Control Problem Constrained by Evolution Equation
}

\author{
O. Olotu \\ Department of Mathematical Sciences, \\ The Federal University of Technology, P.M.B 704, Akure, Nigeria
}

\begin{abstract}
Problem statement: Here, we develop a discretized scheme using only the penalty method without involving the multiplier parameter to examine the convergence and geometric ratio profiles. Approach: This approach reduces computational time arising from less data manipulation. Objectively, we wish to obtain a numerical solution comparing favourably with the analytic solution.. Methodologically, we discretize the given problem, obtain an unconstrained formulation and construct an operator which sets the stage for the application of the discretized extended conjugate gradient method. Results: We analyse the efficiency of the developed scheme by considering an example and examining the generated sequential approximate solutions and the convergence ratio profile computed quadratically per cycle using the discretized conjugate gradient method. Conclusion/Recommendations: Both results, as shown in the table, look comparably and this suggests that the developed scheme may very well approximate an analytic solution of a given problem to an appreciable level of tolerance without its prior knowledge.
\end{abstract}

Key words: Discretized scheme, operator v, evolution equation, examining convergence, Conjugate Gradient Method (CGM), control problem constrained, numerical solution, square integrable, penalty parameter, conjugate gradient algorithm

\section{INTRODUCTION}

Here, we examine a discretized scheme via the penalty method to examine the convergence analysis of optimal control problem constrained by evolution equation with real coefficients.. The constrained problem is converted to an unconstrained problem via the penalty method (Fletcher and Reeves, 1964). Discretization of its time interval and finite difference method for its differential constraint were employed to obtain the discretized formulation of the problem. With this formulation, an associated operator was obtained using the modified extended Conjugate Gradient Method (CGM) (Hestenes, 1969; Ibiejugba and Onumanyi, 1984). Using the modified conjugate gradient method, an example was considered to examine the numerical solution and the convergence analysis as it compares favorably with each other. To this end, a generalized quadratic problem constrained by evolution equation with real coefficients is considered in the next paragraph for our developed scheme.

\section{MATERIALS AND METHODS}

\section{Generalized problem:}

$\operatorname{Minimize} L(t, x, u)_{k}=\int_{0}^{T}\left[\left(p x^{2}+\mathrm{qu}^{2}\right) d t\right]$
Such that:

$$
\begin{aligned}
& a \dot{x}(t-r)+b \dot{x}(t)=c x(t)+d u(t), \\
& x(0)=x_{0}, \dot{x}(t)=h(t), t \in[-r, 0]
\end{aligned}
$$

where, $\mathrm{p}, \mathrm{q}>$, a,b,c are real numbers and $\mathrm{K}$ denotes the Cartesian product of the following two spaces $\mathrm{K}=\mathrm{H}_{1}[0 . \sigma] \times \mathrm{L}_{2}^{\mathrm{q}}[0, \sigma]$, where $\mathrm{H}_{1}[0, \sigma]$ stands for the Sobolev space of the absolutely continuous functions $\mathrm{x}(\bullet)$ such that both $\mathrm{x}(\bullet)$ and $\dot{\mathrm{x}}(\bullet)$ are square integrable over $[0, \sigma]$ and $\mathrm{L}_{2}^{\mathrm{q}}[0, \sigma]$ stands for the Hilbert space consisting of equivalence classes of square integrable functions from $[0, \sigma]-\mathrm{R}^{\mathrm{q}}$.

\section{Remark:}

$\dot{x}(t-r)=\dot{x}(t)\left(\begin{array}{cc}h(s), s \in & {[-r, 0]} \\ \dot{x}(s), s \in & {[0, T-r]}\end{array}\right)$

When $h(t)=0$, then $\dot{x}(t-r)=\dot{x}(t)$

Discretization: By discretizing (1) and (2) using (Fletcher and Reeves, 1964; Glad, 1979) subdivide $[0, \mathrm{~T}]$ into $\mathrm{n}$ equal intervals $\left[\mathrm{t}_{\mathrm{k}}, \mathrm{t}_{\mathrm{k}+1}\right]$ at meshpoints $\mathrm{x}_{0}<\mathrm{x}_{1}<\mathrm{x}_{2},<\ldots<\mathrm{x}_{\mathrm{n}}$ where $\mathrm{n}-1$ is the number of partition 
points chosen arbitrarily, thus having $(\mathrm{n}+1)$ partition points, with $\mathrm{x}_{\mathrm{j}}=\mathrm{j} * \Delta_{\mathrm{j}}, \mathrm{j}=0,1,2, \ldots \ldots \mathrm{n}$ and $\Delta_{\mathrm{j}}=\Delta_{\mathrm{k}}$ is the fixed length of each subinterval for $\mathrm{j}=\mathrm{k}$ or not. By $\mathrm{j}^{*} \Delta_{\mathrm{j}}$, it means $\mathrm{j}$ multiplied by $\Delta_{\mathrm{j}}$. Let $\mathrm{t}_{0}=0$ and $\mathrm{t}_{\mathrm{k}}=\sum_{\mathrm{j}=1}^{\mathrm{k}-1} \Delta \mathrm{j}, \mathrm{k}=1,2,3, \ldots, \mathrm{n}, \mathrm{t}_{\mathrm{n}}=\mathrm{T}$ :

$\mathrm{x}(\mathrm{k})=\mathrm{x}_{\mathrm{k}}\left(\mathrm{t}_{\mathrm{k}}\right), \mathrm{u}_{\mathrm{k}}=\mathrm{u}_{\mathrm{k}}\left(\mathrm{t}_{\mathrm{k}}\right), \mathrm{k}=0,1,2, \ldots, \mathrm{n}$

By Euler's scheme or finite difference method:

$\dot{\mathrm{x}}(\mathrm{k})=(\mathrm{x}(\mathrm{k}+1)-\mathrm{x}(\mathrm{k})) / \Delta_{\mathrm{k}}, \mathrm{k}=0,1,2, \ldots \mathrm{n}-1$

the generalized problem (1) becomes.

\section{Generalized problem 3.1:}

$\operatorname{Minimize~} \mathrm{j}\left(\mathrm{t}_{\mathrm{k}}, \mathrm{x}_{\mathrm{k}}, \mathrm{u}_{\mathrm{k}}\right)=\sum_{\mathrm{k}=0}^{\mathrm{n}} \Delta_{\mathrm{k}}\left(\mathrm{x}_{\mathrm{k}}\left(\mathrm{t}_{\mathrm{k}}\right)\right.$

Subject to Eq. 3 and 4:

$\mathrm{a}\left(\mathrm{x}_{\mathrm{k}+1}-\left(\mathrm{t}_{\mathrm{k}+1}-\gamma_{\mathrm{k}+1}\right)-\mathrm{x}_{\mathrm{k}}\left(\mathrm{t}_{\mathrm{k}}-\gamma_{\mathrm{k}}\right)\right) / \Delta_{\mathrm{k}}+\mathrm{b}\left(\mathrm{x}_{\mathrm{k}+1}\left(\mathrm{t}_{\mathrm{k}+1}\right)\right.$

$\left.-\mathrm{x}_{\mathrm{k}}\left(\mathrm{t}_{\mathrm{k}}\right)\right) / \Delta_{\mathrm{k}}=\mathrm{cx}_{\mathrm{k}}\left(\mathrm{t}_{\mathrm{k}}\right)+\mathrm{du}_{\mathrm{k}}\left(\mathrm{t}_{\mathrm{k}}\right) \mathrm{x}_{\mathrm{k}}(0)=0$

\section{Application of the penalty parameter:}

$\operatorname{Minimize~} \mathrm{j}\left(\mathrm{t}_{\mathrm{k}}, \mathrm{x}_{\mathrm{k}}, \mathrm{u}_{\mathrm{k}}\right)=\sum_{\mathrm{k}=0}^{\mathrm{n}} \Delta_{\mathrm{k}}\left(\mathrm{x}_{\mathrm{k}}\left(\mathrm{t}_{\mathrm{k}}\right)\right.$

Such that:

$\mu\left[\operatorname{ax}_{\mathrm{k}+1}\left(\mathrm{t}_{\mathrm{k}}-\gamma_{\mathrm{k}}\right)-\mathrm{ax}_{\mathrm{k}}\left(\mathrm{t}_{\mathrm{k}}\right)+\mathrm{bx}_{\mathrm{k}+1}\left(\mathrm{t}_{\mathrm{k}}\right)-\Delta_{\mathrm{k}} \mathrm{cx}_{\mathrm{k}}-\Delta_{\mathrm{k}} \mathrm{du}_{\mathrm{k}}\right]^{2}$

Now (5) and (6) become the following using (Di Pillo and Grippo, 1979; Glad, 1979) Eq. 7:

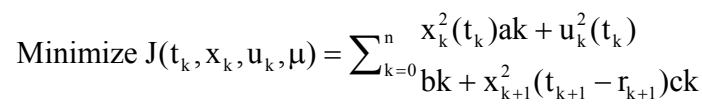

$+\mathrm{x}_{\mathrm{k}+1}\left(\mathrm{t}_{\mathrm{k}+1}-\mathrm{r}_{\mathrm{k}+1}\right) \mathrm{x}_{\mathrm{k}}\left(\mathrm{t}_{\mathrm{k}}-\mathrm{r}_{\mathrm{k}}\right) \mathrm{ck} 1+\mathrm{x}_{\mathrm{k}+1}\left(\mathrm{t}_{\mathrm{k}+1}-\mathrm{r}_{\mathrm{k}+1}\right) \mathrm{x}_{\mathrm{k}+1}\left(\mathrm{t}_{\mathrm{k}+1}\right) \mathrm{ck} 2$

$+\mathrm{x}_{\mathrm{k}+1}\left(\mathrm{t}_{\mathrm{k}+1}-\mathrm{r}_{\mathrm{k}+1}\right) \mathrm{x}_{\mathrm{k}}\left(\mathrm{t}_{\mathrm{k}}-\mathrm{r}_{\mathrm{k}}\right) \mathrm{pk}+\mathrm{x}_{\mathrm{k}+1}\left(\mathrm{t}_{\mathrm{k}+1}-\mathrm{r}_{\mathrm{k}+1}\right) \mathrm{u}_{\mathrm{k}}\left(\mathrm{t}_{\mathrm{k}}\right) \mathrm{mk}$

$+\mathrm{x}_{\mathrm{k}}\left(\mathrm{t}_{\mathrm{k}}-\mathrm{r}_{\mathrm{k}}\right) \mathrm{x}_{\mathrm{k}+1}\left(\mathrm{t}_{\mathrm{k}+1}\right) \mathrm{mk} 2+\mathrm{x}_{\mathrm{k}}\left(\mathrm{t}_{\mathrm{k}}-\mathrm{r}_{\mathrm{k}}\right) \mathrm{x}_{\mathrm{k}}\left(\mathrm{t}_{\mathrm{k}}\right) \mathrm{nk}$

$+x_{k}\left(t_{k}-r_{k}\right) u_{k}\left(t_{k}\right) w k+x_{k+1}^{2}\left(t_{k+1}\right) w k 1+x_{k+1}\left(t_{k+1}\right) x_{k}\left(t_{k}\right) v_{k}$

$+\mathrm{x}_{\mathrm{k}+1}\left(\mathrm{t}_{\mathrm{k}+1}\right) \mathrm{u}_{\mathrm{k}}\left(\mathrm{t}_{\mathrm{k}}\right) \mathrm{q}_{\mathrm{k}}+\mathrm{x}_{\mathrm{k}}\left(\mathrm{t}_{\mathrm{k}}\right) \mathrm{u}_{\mathrm{k}}\left(\mathrm{t}_{\mathrm{k}}\right) \mathrm{dk} 1$

Where:

$$
\begin{aligned}
& \text { ak }=\mathrm{p} \Delta_{\mathrm{k}}+\mathrm{b}^{2} \mu+2 \mathrm{bc} \Delta_{\mathrm{k}} \mu+\Delta_{\mathrm{k}}^{2} \mathrm{c}^{2} \mu \\
& \mathrm{bk}=\mathrm{q} \Delta_{\mathrm{k}}+\mathrm{u} \Delta_{\mathrm{k}}^{2} \mathrm{~d}^{2} \\
& \mathrm{ck}=\mu \mathrm{a}^{2} \\
& \mathrm{ck} 1=-2 \mu \mathrm{a}^{2}
\end{aligned}
$$

$$
\begin{aligned}
& \mathrm{ck} 2=2 \mu \mathrm{ab} \\
& \mathrm{pk}=-2 \mathrm{ab} \mu-2 \mathrm{ac} \Delta_{\mathrm{k}} \\
& \mathrm{mk}=-2 \mathrm{ad} \Delta_{\mathrm{k}} \mu \\
& \mathrm{mk} 1=\mu \mathrm{a}^{2} \\
& \mathrm{mk} 2=-2 \mu \mathrm{ab} \\
& \mathrm{nk}=2 \mu \mathrm{ab}+2 \mu \mathrm{ac} \Delta_{\mathrm{k}} \\
& \mathrm{wk}=2 \mu \mathrm{ad} \Delta_{\mathrm{k}} \\
& \mathrm{wk} 1=\mu \mathrm{b}^{2} \\
& \mathrm{vk}=-2 \mu \mathrm{b}^{2} \\
& \mathrm{qk}=-2 \mu \mathrm{bd} \Delta_{\mathrm{k}} \\
& \mathrm{dk} 1=2 \mu \mathrm{bd} \Delta_{\mathrm{k}}+2 \mu \mathrm{cd} \Delta_{\mathrm{k}}^{2}
\end{aligned}
$$

Setting $\mathrm{y}_{\mathrm{k}}=\mathrm{x}_{\mathrm{k}+1}\left(\mathrm{t}_{\mathrm{k}+1}\right)$ in Eq. 7 we have Eq. 8:

$$
\begin{aligned}
& \text { Minimize } J\left(t_{k}, x_{k}, u_{k}, \mu\right)=\sum_{k=0}^{n}\left(x_{k}^{2}\left(t_{k}\right) a k+u_{k}^{2}\left(t_{k}\right) b k+y_{k}^{2}\left(t_{k}-r_{k}\right)\right. \\
& +y_{k}\left(t_{k}\right) x_{k}\left(t_{k}-r_{k}\right) y_{k}\left(t_{k}\right) c k 2+y_{k}\left(t_{k}-r_{k}\right) x_{k} \\
& \left(t_{k}\right) p k+y_{k}\left(t_{k}-r_{k}\right) u_{k}\left(t_{k}\right) m k \\
& +x_{k}^{2}\left(t_{k}-r_{k}\right) m k 1+x_{k}\left(t_{k}-r_{k}\right) y_{k}\left(t_{k+1}\right) m k 2 \\
& +x_{k}\left(t_{k}-r_{k}\right) x_{k}\left(t_{k}\right) n k \\
& +x_{k}\left(t_{k}-r_{k}\right) u_{k}\left(t_{k}\right) w k+y_{k}^{2}\left(t_{k}\right) w k 1+y_{k}\left(t_{k}\right) x_{k} \\
& \left(t_{k}\right) v_{k}+y_{k}\left(t_{k}\right) u_{k}\left(t_{k}\right) q k+x_{k}\left(t_{k}\right) u_{k}\left(t_{k}\right) d k 1
\end{aligned}
$$

Construction of operator v: We now construct the operator $\mathrm{V}$ for the conjugate gradient method. The bilinear form associated with (7) using (Ibiejugba and Onumanyi, 1984) is given as Eq. 9 and 10:

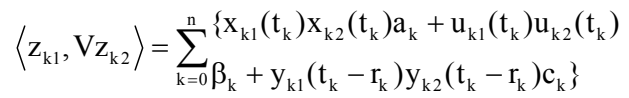

$$
\begin{aligned}
& +\mathrm{y}_{\mathrm{k} 1}\left(\mathrm{t}_{\mathrm{k}}-\mathrm{r}_{\mathrm{k}}\right) \mathrm{x}_{\mathrm{k} 2}\left(\mathrm{t}_{\mathrm{k}}-\mathrm{r}_{\mathrm{k}}\right) \mathrm{c}_{\mathrm{k} 1}+\mathrm{y}_{\mathrm{k} 2}\left(\mathrm{t}_{\mathrm{k}}-\mathrm{r}_{\mathrm{k}}\right) \mathrm{x}_{\mathrm{k} 1}\left(\mathrm{t}_{\mathrm{k}}-\mathrm{r}_{\mathrm{k}}\right) \\
& \mathrm{c}_{\mathrm{k} 1}+\mathrm{y}_{\mathrm{K} 1}\left(\mathrm{t}_{\mathrm{k}}-\mathrm{r}_{\mathrm{k}}\right) \mathrm{y}_{\mathrm{k} 2}\left(\mathrm{t}_{\mathrm{k}}\right) \mathrm{c}_{\mathrm{k} 2} \\
& +\mathrm{y}_{\mathrm{k} 2}\left(\mathrm{t}_{\mathrm{k}}-\mathrm{r}_{\mathrm{k}}\right) \mathrm{y}_{\mathrm{k} 1}\left(\mathrm{t}_{\mathrm{k}}\right) \mathrm{c}_{\mathrm{k} 2}+\mathrm{y}_{\mathrm{k} 1}\left(\mathrm{t}_{\mathrm{k}}-\mathrm{r}_{\mathrm{k}}\right) \mathrm{x}_{\mathrm{k} 2}\left(\mathrm{t}_{\mathrm{k}}\right) \\
& \mathrm{p}_{\mathrm{k}}+\mathrm{y}_{\mathrm{k} 2}\left(\mathrm{t}_{\mathrm{k}}-\mathrm{r}_{\mathrm{k}}\right) \mathrm{x}_{\mathrm{k} 1}\left(\mathrm{t}_{\mathrm{k}}\right) \mathrm{p}_{\mathrm{k}} \\
& +y_{k 1}\left(t_{k}-r_{k}\right) u_{k 2}\left(t_{k}\right) m_{k}+y_{k 2}\left(t_{k}-r_{k}\right) u_{k 1}\left(t_{k}\right) \\
& \mathrm{m}_{\mathrm{k}}+\mathrm{x}_{\mathrm{k} 1}\left(\mathrm{t}_{\mathrm{k}}-\mathrm{r}_{\mathrm{k}}\right) \mathrm{x}_{\mathrm{k} 2}\left(\mathrm{t}_{\mathrm{k}}-\mathrm{r}_{\mathrm{k}}\right) \mathrm{m}_{\mathrm{k} 1} \\
& +\mathrm{x}_{\mathrm{k} 1}\left(\mathrm{t}_{\mathrm{k}}-\mathrm{r}_{\mathrm{k}}\right) \mathrm{y}_{\mathrm{k} 2}\left(\mathrm{t}_{\mathrm{k}}\right) \mathrm{m}_{\mathrm{k} 2}+\mathrm{x}_{\mathrm{k} 2}\left(\mathrm{t}_{\mathrm{k}}-\mathrm{r}_{\mathrm{k}}\right) \mathrm{y}_{\mathrm{k} 1}\left(\mathrm{t}_{\mathrm{k}}\right) \\
& \mathrm{m}_{\mathrm{k} 2}+\mathrm{x}_{\mathrm{k} 1}\left(\mathrm{t}_{\mathrm{k}}-\mathrm{r}_{\mathrm{k}}\right) \mathrm{x}_{\mathrm{k} 2}\left(\mathrm{t}_{\mathrm{k}}\right) \mathrm{n}_{\mathrm{k}} \\
& +x_{k 2}\left(t_{k}-r_{k}\right) x_{k 1}\left(t_{k}\right) n_{k}+x_{k 1}\left(t_{k}-r_{k}\right) u_{k}\left(t_{k}\right) \\
& \mathrm{w}_{\mathrm{k}}+\mathrm{x}_{\mathrm{k} 2}\left(\mathrm{t}_{\mathrm{k}}-\mathrm{r}_{\mathrm{k}}\right) \mathrm{u}_{\mathrm{k} 1}\left(\mathrm{t}_{\mathrm{k}}\right) \mathrm{w}_{\mathrm{k}} \\
& +\mathrm{y}_{\mathrm{k} 1}\left(\mathrm{t}_{\mathrm{k}}\right) \mathrm{y}_{\mathrm{k} 2}\left(\mathrm{t}_{\mathrm{k}}\right) \mathrm{w}_{\mathrm{k} 1}+\mathrm{y}_{\mathrm{k} 1}\left(\mathrm{t}_{\mathrm{k}}\right) \mathrm{x}_{\mathrm{k} 2}\left(\mathrm{t}_{\mathrm{k}}\right) \mathrm{v}_{\mathrm{k}}+\mathrm{y}_{\mathrm{k} 2} \\
& \left(t_{k}\right) x_{k 1}\left(t_{k}\right) v_{k}+y_{k 1}\left(t_{k}\right) u_{k 2}\left(t_{k}\right) q_{k} \\
& +\mathrm{y}_{\mathrm{k} 2}\left(\mathrm{t}_{\mathrm{k}}\right) \mathrm{u}_{\mathrm{k} 1}\left(\mathrm{t}_{\mathrm{k}}\right) \mathrm{q}_{\mathrm{k}}+\mathrm{x}_{\mathrm{k} 1}\left(\mathrm{t}_{\mathrm{k}}\right) \mathrm{u}_{\mathrm{k} 2}\left(\mathrm{t}_{\mathrm{k}}\right) \\
& \mathrm{d}_{\mathrm{k}}+\mathrm{x}_{\mathrm{k} 2}\left(\mathrm{t}_{\mathrm{k}}\right) \mathrm{u}_{\mathrm{k} 1}\left(\mathrm{t}_{\mathrm{k}}\right) \mathrm{d}_{\mathrm{k}}
\end{aligned}
$$




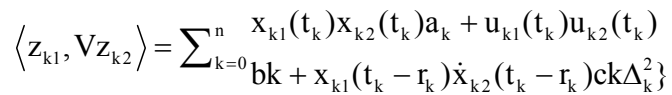

$$
\begin{aligned}
& +\dot{x}_{\mathrm{K} 1}\left(\mathrm{t}_{\mathrm{k}}-\mathrm{r}_{\mathrm{k}}\right) \mathrm{x}_{\mathrm{k} 2}\left(\mathrm{t}_{\mathrm{k}}-\mathrm{r}_{\mathrm{k}}\right) \Delta_{\mathrm{k}} \mathrm{ck}+\mathrm{x}_{\mathrm{k} 1}\left(\mathrm{t}_{\mathrm{k}}-\mathrm{r}_{\mathrm{k}}\right) \dot{\mathrm{x}}_{\mathrm{k} 2}\left(\mathrm{t}_{\mathrm{k}}-\mathrm{r}_{\mathrm{k}}\right) \Delta_{\mathrm{k}} \mathrm{ck} \\
& +\mathrm{x}_{\mathrm{K} 1}\left(\mathrm{t}_{\mathrm{k}}-\mathrm{r}_{\mathrm{k}}\right) \mathrm{x}_{\mathrm{k} 2}\left(\mathrm{t}_{\mathrm{k}}-\mathrm{r}_{\mathrm{k}}\right) \mathrm{ck}+\dot{\mathrm{x}}_{\mathrm{k} 1}\left(\mathrm{t}_{\mathrm{k}}-\mathrm{r}_{\mathrm{k}}\right) \mathrm{x}_{\mathrm{k} 2}\left(\mathrm{t}_{\mathrm{k}}-\mathrm{r}_{\mathrm{k}}\right) \Delta_{\mathrm{k}} \mathrm{ck} 1 \\
& +\mathrm{x}_{\mathrm{K} 1}\left(\mathrm{t}_{\mathrm{k}}-\mathrm{r}_{\mathrm{k}}\right) \mathrm{x}_{\mathrm{k} 2}\left(\mathrm{t}_{\mathrm{k}}-\mathrm{r}_{\mathrm{k}}\right) \mathrm{ck} 1+\dot{\mathrm{x}}_{\mathrm{k}} \mathrm{k} 2\left(\mathrm{t}_{\mathrm{k}}-\mathrm{r}_{\mathrm{k}}\right) \dot{\mathrm{x}}_{\mathrm{k} 1}\left(\mathrm{t}_{\mathrm{k}}-\mathrm{r}_{\mathrm{k}}\right) \Delta_{\mathrm{k}} \mathrm{ck} 1 \\
& +\mathrm{x}_{\mathrm{K} 2}\left(\mathrm{t}_{\mathrm{k}}-\mathrm{r}_{\mathrm{k}}\right) \mathrm{x}_{\mathrm{k} 1}\left(\mathrm{t}_{\mathrm{k}}-\mathrm{r}_{\mathrm{k}}\right) \mathrm{ck} 1+\dot{\mathrm{x}}_{\mathrm{k} 1}\left(\mathrm{t}_{\mathrm{k}}-\mathrm{r}_{\mathrm{k}}\right) \dot{\mathrm{x}}_{\mathrm{k} 2} \\
& \left(\mathrm{t}_{\mathrm{k}}\right) \Delta_{\mathrm{k}} \mathrm{ck} 2+\dot{\mathrm{x}}_{\mathrm{k} 1}\left(\mathrm{t}_{\mathrm{k}}-\mathrm{r}_{\mathrm{k}}\right) \dot{\mathrm{x}}_{\mathrm{k} 2}\left(\mathrm{t}_{\mathrm{k}}\right) \Delta_{\mathrm{k}} \mathrm{ck} 2 \\
& +\mathrm{x}_{\mathrm{K} 1}\left(\mathrm{t}_{\mathrm{k}}-\mathrm{r}_{\mathrm{k}}\right) \dot{\mathrm{x}}_{\mathrm{k} 2}\left(\mathrm{t}_{\mathrm{k}}\right) \Delta_{\mathrm{k}} \mathrm{ck} 2+\mathrm{x}_{\mathrm{k} 1}\left(\mathrm{t}_{\mathrm{k}}-\mathrm{r}_{\mathrm{k}}\right) \mathrm{x}_{\mathrm{k} 2}\left(\mathrm{t}_{\mathrm{k}}\right) \\
& \mathrm{ck} 2+\dot{\mathrm{x}}_{\mathrm{k} 2}\left(\mathrm{t}_{\mathrm{k}}-\mathrm{r}_{\mathrm{k}}\right) \dot{\mathrm{x}}_{\mathrm{k} 1}\left(\mathrm{t}_{\mathrm{k}}\right) \Delta_{\mathrm{k}}^{2} \mathrm{ck} 2 \\
& +\dot{\mathrm{x}}_{\mathrm{K} 2}\left(\mathrm{t}_{\mathrm{k}}-\mathrm{r}_{\mathrm{k}}\right) \mathrm{x}_{\mathrm{k} 1}\left(\mathrm{t}_{\mathrm{k}}\right) \Delta_{2} \mathrm{ck} 2+\mathrm{x}_{\mathrm{k} 2}\left(\mathrm{t}_{\mathrm{k}}-\mathrm{r}_{\mathrm{k}}\right) \dot{\mathrm{x}}_{\mathrm{k} 1}\left(\mathrm{t}_{\mathrm{k}}\right) \\
& \Delta_{\mathrm{k}} \mathrm{ck} 2+\mathrm{x}_{\mathrm{k} 2}\left(\mathrm{t}_{\mathrm{k}}-\mathrm{r}_{\mathrm{k}}\right) \mathrm{x}_{\mathrm{k} 1}\left(\mathrm{t}_{\mathrm{k}}\right) \operatorname{ck} 2 \\
& +\dot{\mathrm{x}}_{\mathrm{K} 1}\left(\mathrm{t}_{\mathrm{k}}-\mathrm{r}_{\mathrm{k}}\right) \mathrm{x}_{\mathrm{k} 2}\left(\mathrm{t}_{\mathrm{k}}\right) \Delta_{\mathrm{k}} \mathrm{pk} 2+\mathrm{x}_{\mathrm{k} 1}\left(\mathrm{t}_{\mathrm{k}}-\mathrm{r}_{\mathrm{k}}\right) \mathrm{x}_{\mathrm{k} 2}\left(\mathrm{t}_{\mathrm{k}}\right) \\
& \mathrm{pk}+\dot{\mathrm{x}}_{\mathrm{k} 2}\left(\mathrm{t}_{\mathrm{k}}-\mathrm{r}_{\mathrm{k}}\right) \mathrm{x}_{\mathrm{k} 1}\left(\mathrm{t}_{\mathrm{k}}\right) \Delta_{\mathrm{k}} \mathrm{pk} \\
& +\mathrm{x}_{\mathrm{K} 2}\left(\mathrm{t}_{\mathrm{k}}-\mathrm{r}_{\mathrm{k}}\right) \mathrm{x}_{\mathrm{k} 1}\left(\mathrm{t}_{\mathrm{k}}\right) \mathrm{pk}+\dot{\mathrm{x}}_{\mathrm{k} 1}\left(\mathrm{t}_{\mathrm{k}}-\mathrm{r}_{\mathrm{k}}\right) \mathrm{u}_{\mathrm{k} 2}\left(\mathrm{t}_{\mathrm{k}}\right) \\
& \Delta_{\mathrm{k}} \mathrm{mk}+\mathrm{x}_{\mathrm{k} 1}\left(\mathrm{t}_{\mathrm{k}}-\mathrm{r}_{\mathrm{k}}\right) \mathrm{u}_{\mathrm{k} 2}\left(\mathrm{t}_{\mathrm{k}}\right) \mathrm{mk} \\
& \dot{\mathrm{x}}_{\mathrm{K} 2}\left(\mathrm{t}_{\mathrm{k}}-\mathrm{r}_{\mathrm{k}}\right) \mathrm{u}_{\mathrm{k} 1}\left(\mathrm{t}_{\mathrm{k}}\right) \Delta_{\mathrm{k}} \mathrm{mk}+\mathrm{x}_{\mathrm{k} 2}\left(\mathrm{t}_{\mathrm{k}}-\mathrm{r}_{\mathrm{k}}\right) \mathrm{u}_{\mathrm{k} 1}\left(\mathrm{t}_{\mathrm{k}}\right) \\
& m k+x_{k 1}\left(t_{k}-r_{k}\right) x_{k 2}\left(t_{k}-r_{k}\right) m k 1 \\
& +\mathrm{x}_{\mathrm{K} 1}\left(\mathrm{t}_{\mathrm{k}}-\mathrm{r}_{\mathrm{k}}\right) \dot{\mathrm{x}}_{\mathrm{k} 2}\left(\mathrm{t}_{\mathrm{k}}\right) \mathrm{nk}+\mathrm{x}_{\mathrm{k} 2}\left(\mathrm{t}_{\mathrm{k}}-\mathrm{r}_{\mathrm{k}}\right) \mathrm{x}_{\mathrm{k} 1}\left(\mathrm{t}_{\mathrm{k}}\right) \\
& \mathrm{mk} 2+\mathrm{x}_{\mathrm{k} 2}\left(\mathrm{t}_{\mathrm{k}}-\mathrm{r}_{\mathrm{k}}\right) \dot{\mathrm{x}}_{\mathrm{k} 1}\left(\mathrm{t}_{\mathrm{k}}\right) \Delta_{\mathrm{k}} \mathrm{mk} 2 \\
& +\mathrm{x}_{\mathrm{K} 2}\left(\mathrm{t}_{\mathrm{k}}-\mathrm{r}_{\mathrm{k}}\right) \mathrm{x}_{\mathrm{k} 1}\left(\mathrm{t}_{\mathrm{k}}\right) \mathrm{mk} 2+\mathrm{x}_{\mathrm{k} 1}\left(\mathrm{t}_{\mathrm{k}}-\mathrm{r}_{\mathrm{k}}\right) \mathrm{x}_{\mathrm{k} 2}\left(\mathrm{t}_{\mathrm{k}}\right) \\
& \mathrm{nk}+\mathrm{x}_{\mathrm{k} 2}\left(\mathrm{t}_{\mathrm{k}}-\mathrm{r}_{\mathrm{k}}\right) \mathrm{x}_{\mathrm{k} 1}\left(\mathrm{t}_{\mathrm{k}}\right) \mathrm{nk} \\
& +\mathrm{x}_{\mathrm{K} 1}\left(\mathrm{t}_{\mathrm{k}}-\mathrm{r}_{\mathrm{k}}\right) \mathrm{u}_{\mathrm{k} 2}\left(\mathrm{t}_{\mathrm{k}}\right) \mathrm{wk}+\mathrm{x}_{\mathrm{k} 2}\left(\mathrm{t}_{\mathrm{k}}-\mathrm{r}_{\mathrm{k}}\right) \\
& \mathrm{u}_{\mathrm{k} 1}\left(\mathrm{t}_{\mathrm{k}}\right) \mathrm{wk}+\dot{\mathrm{x}}_{\mathrm{k} 1}\left(\mathrm{t}_{\mathrm{k}}\right) \dot{\mathrm{x}}_{\mathrm{k} 2}\left(\mathrm{t}_{\mathrm{k}}\right) \Delta_{\mathrm{k}} \mathrm{wk} 1 \\
& +\dot{\mathrm{x}}_{\mathrm{K} 1}\left(\mathrm{t}_{\mathrm{k}}-\mathrm{r}_{\mathrm{k}}\right) \mathrm{x}_{\mathrm{k} 2}\left(\mathrm{t}_{\mathrm{k}}\right) \mathrm{wk} 1+\mathrm{x}_{\mathrm{k} 1}\left(\mathrm{t}_{\mathrm{k}}\right) \dot{\mathrm{x}}_{\mathrm{k} 2}\left(\mathrm{t}_{\mathrm{k}}\right) \\
& \Delta_{\mathrm{k}} \mathrm{wk} 1+\mathrm{x}_{\mathrm{k} 1}\left(\mathrm{t}_{\mathrm{k}}\right) \mathrm{x}_{\mathrm{k} 2}\left(\mathrm{t}_{\mathrm{k}}\right) \mathrm{w}_{\mathrm{k} 1} \\
& +\dot{\mathrm{x}}_{\mathrm{K} 1}\left(\mathrm{t}_{\mathrm{k}}\right) \mathrm{x}_{\mathrm{k} 2}\left(\mathrm{t}_{\mathrm{k}}\right) \Delta_{\mathrm{k}} \mathrm{vk}+\mathrm{x}_{\mathrm{k} 1}\left(\mathrm{t}_{\mathrm{k}}\right) \mathrm{x}_{\mathrm{k} 2}\left(\mathrm{t}_{\mathrm{k}}\right) \\
& \mathrm{vk}+\dot{\mathrm{x}}_{\mathrm{k} 2}\left(\mathrm{t}_{\mathrm{k}}\right) \mathrm{x}_{\mathrm{k} 1}\left(\mathrm{t}_{\mathrm{k}}\right) \mathrm{vk} \Delta_{\mathrm{k}} \\
& +\mathrm{x}_{\mathrm{K} 2}\left(\mathrm{t}_{\mathrm{k}}\right) \mathrm{x}_{\mathrm{k} 1}\left(\mathrm{t}_{\mathrm{k}}\right) \mathrm{vk}+\dot{\mathrm{x}}_{\mathrm{k} 1}\left(\mathrm{t}_{\mathrm{k}}\right) \mathrm{u}_{\mathrm{k} 2}\left(\mathrm{t}_{\mathrm{k}}\right) \\
& \Delta_{\mathrm{k}} \mathrm{qk}+\mathrm{x}_{\mathrm{k} 1}\left(\mathrm{t}_{\mathrm{k}}\right) \mathrm{u}_{\mathrm{k} 2}\left(\mathrm{t}_{\mathrm{k}}\right) \mathrm{qk}+\dot{\mathrm{x}}_{\mathrm{k} 2}\left(\mathrm{t}_{\mathrm{k}}\right) \mathrm{u}_{\mathrm{k} 2}\left(\mathrm{t}_{\mathrm{k}}\right) \Delta_{\mathrm{k}} \mathrm{qk} \\
& +\mathrm{x}_{\mathrm{K} 2}\left(\mathrm{t}_{\mathrm{k}}\right) \mathrm{u}_{\mathrm{k} 1}\left(\mathrm{t}_{\mathrm{k}}\right) \mathrm{qk}+\mathrm{x}_{\mathrm{k} 1}\left(\mathrm{t}_{\mathrm{k}}\right) \\
& \mathrm{u}_{\mathrm{k} 2}\left(\mathrm{t}_{\mathrm{k}}\right) \mathrm{dk}+\mathrm{x}_{\mathrm{k} 2}\left(\mathrm{t}_{\mathrm{k}}\right) \mathrm{u}_{\mathrm{k} 1}\left(\mathrm{t}_{\mathrm{k}}\right) \mathrm{dk}
\end{aligned}
$$

Now, we state the Theorem establishing operator V and provide a proof;

Theorem 5.1 Let the initial guess of the conjugate gradient algorithm be $\mathrm{z}_{0}\left(\mathrm{t}_{\mathrm{k}}\right)$ so that $\mathrm{z}_{0}\left(\mathrm{t}_{\mathrm{k}}\right)=\left(\mathrm{x}_{0}\left(\mathrm{t}_{\mathrm{k}}\right), \mathrm{u}_{0}\left(\mathrm{t}_{\mathrm{k}}\right), \mathrm{h}-\right.$ ${ }_{0}\left(t_{k}\right)$ ), then the control operator $\mathrm{V}$ associated with the generalized problem is given by Eq. 11:

$$
\mathrm{Vz}_{\mathrm{k} 2}\left(\mathrm{t}_{\mathrm{k}}\right)=\left(\begin{array}{ccc}
\mathrm{V}_{11} & \mathrm{~V}_{12} & \mathrm{~V}_{13} \\
\mathrm{~V}_{21} & \mathrm{~V}_{22} & \mathrm{~V}_{23} \\
\mathrm{~V}_{31} & \mathrm{~V}_{32} & \mathrm{~V}_{33}
\end{array}\right)\left(\begin{array}{l}
\mathrm{x}_{\mathrm{k} 2}\left(\mathrm{t}_{\mathrm{k}}\right) \\
\mathrm{u}_{\mathrm{k} 2}\left(\mathrm{t}_{\mathrm{k}}\right) \\
\mathrm{h}_{\mathrm{k} 2}\left(\mathrm{t}_{\mathrm{k}}\right)
\end{array}\right)
$$

where, $\mathrm{z}_{\mathrm{k} 2}\left(\mathrm{t}_{\mathrm{k}}\right)=\left(\mathrm{x}_{\mathrm{k} 2}\left(\mathrm{t}_{\mathrm{k}}\right), \mathrm{u}_{\mathrm{k} 2}\left(\mathrm{t}_{\mathrm{k}}\right) \mathrm{h}_{\mathrm{k} 2}\left(\mathrm{t}_{\mathrm{k}}\right)\right)$

Proof: Find $\mathrm{x}_{\mathrm{k} 2}\left(\mathrm{t}_{\mathrm{k}}\right)$ by setting $\mathrm{u}_{\mathrm{k} 2}\left(\mathrm{t}_{\mathrm{k}}\right)=\mathrm{h}_{\mathrm{k} 2}\left(\mathrm{t}_{\mathrm{k}}\right)=0$ in (5.2) and noting the fact that if $h_{k 2}\left(t_{k}\right)=0$ then $\dot{\mathrm{x}}_{\mathrm{k} 2}\left(\mathrm{t}_{\mathrm{k}}-\gamma_{\mathrm{k}}\right)=\dot{\mathrm{x}}_{\mathrm{k} 2}=\left(\mathrm{t}_{\mathrm{k} 2}\right)$. So Eq. 10 becomes:

$$
\begin{aligned}
& \left\langle\mathrm{z}_{\mathrm{k} 1}, \mathrm{Vz}_{\mathrm{k} 2}\right\rangle=\sum_{\mathrm{k}=0}^{\mathrm{n}} \mathrm{x}_{\mathrm{k} 1}\left(\mathrm{t}_{\mathrm{k}}\right)\left[\mathrm{x}_{\mathrm{k} 2}\left(\mathrm{t}_{\mathrm{k}}\right)\right. \\
& \left.\left(\mathrm{a}_{\mathrm{k}}, \mathrm{ck} 2+\mathrm{pk}+\mathrm{wk} 2+\mathrm{nk}+\mathrm{wk} 1+2 \mathrm{vk}\right)\right] \\
& +\dot{\mathrm{x}}_{\mathrm{k} 2}\left(\mathrm{t}_{\mathrm{k}}\right)\left(\Delta_{\mathrm{k}} \mathrm{ck} 2+\Delta_{\mathrm{k}} \mathrm{pk}+\mathrm{wk} 1+\mathrm{vk}\right) \\
& +2 \mathrm{x}_{\mathrm{k} 2}\left(\mathrm{t}_{\mathrm{k}}+\mathrm{r}_{\mathrm{k}}\right)(\mathrm{ck}+2 \mathrm{ck} 1+\mathrm{ck} 2+\mathrm{pk}+\mathrm{mk} 1+\mathrm{mk} 2+\mathrm{nk}) \\
& \left.+2 \dot{\mathrm{x}}_{\mathrm{k} 2}\left(\mathrm{t}_{\mathrm{k}}+\mathrm{r}_{\mathrm{k}}\right)\left(\Delta_{\mathrm{k}}(\mathrm{ck}+\mathrm{ck} 1+\mathrm{ck} 2+\mathrm{mk} 2)\right)\right] \\
& +\dot{\mathrm{x}}_{\mathrm{k} 1}\left(\mathrm{t}_{\mathrm{k}}\right)\left[\dot { \mathrm { x } } _ { \mathrm { k } 2 } ( \mathrm { t } _ { \mathrm { k } } ) \left(\Delta_{\mathrm{k}}^{2}(\mathrm{ck} 2+\mathrm{wk} 1)\right.\right. \\
& +\mathrm{x}_{\mathrm{k} 2}\left(\mathrm{t}_{\mathrm{k}}\right)\left[\Delta_{\mathrm{k}}\left(\mathrm{ck} 2+\mathrm{mk} 2+\mathrm{v}_{\mathrm{k}}+\mathrm{wk} 1\right)\right. \\
& +\dot{\mathrm{x}}_{\mathrm{k} 2}\left(\mathrm{t}_{\mathrm{k}}+\mathrm{r}_{\mathrm{k}}\right)\left(\Delta_{\mathrm{k}}^{2}(\mathrm{ck}+\mathrm{ck} 2)\right. \\
& +\mathrm{x}_{\mathrm{k} 2}\left(\mathrm{t}_{\mathrm{k}}-\mathrm{r}_{\mathrm{k}}\right)\left(\Delta_{\mathrm{k}}(\mathrm{ck}+\mathrm{ck} 1+\mathrm{ck} 2+\mathrm{pk})\right) \\
& +\mathrm{u}_{\mathrm{k} 1}\left(\mathrm{t}_{\mathrm{k}}\right)\left[\mathrm{x}_{\mathrm{k} 2}\left(\mathrm{t}_{\mathrm{k}}\right)(\mathrm{mk}+\mathrm{wk}+\mathrm{qk}+\mathrm{dk})\right] \\
& +\dot{\mathrm{x}}_{\mathrm{k} 2}\left(\mathrm{t}_{\mathrm{k}}\right)\left(\Delta_{\mathrm{k}}(\mathrm{mk}+\mathrm{qk})\right)+\mathrm{h}_{\mathrm{k} 1}\left(\mathrm{t}_{\mathrm{k}}+\gamma \mathrm{r}_{\mathrm{k}}\right) \\
& \left(\Delta_{\mathrm{k}}(\mathrm{ck}+\mathrm{ck} 1+\mathrm{ck} 2+\mathrm{pk})\right) \\
& +\dot{\mathrm{x}}_{\mathrm{k} 2}\left(\mathrm{t}_{\mathrm{k}}+\mathrm{r}_{\mathrm{k}}\right)\left(\Delta_{\mathrm{k}}^{2}(\mathrm{ck}+\mathrm{ck} 2)\right)
\end{aligned}
$$

For further compactness, (12) becomes (13):

$$
\begin{aligned}
& \left\langle\mathrm{z}_{\mathrm{k} 1}, \mathrm{Vz}_{\mathrm{k} 2}\right\rangle=\sum_{\mathrm{k}=0}^{\mathrm{n}} \begin{array}{l}
\mathrm{x}_{\mathrm{k} 1}\left(\mathrm{t}_{\mathrm{k}}\right)\left[\mathrm{x}_{\mathrm{k} 2}\left(\mathrm{t}_{\mathrm{k}}\right) \mathrm{Nk} 1+\dot{\mathrm{x}}_{\mathrm{k} 2}\left(\mathrm{t}_{\mathrm{k}}\right)\right. \\
\mathrm{Nk} 2+2 \mathrm{x}_{\mathrm{k} 2}\left(\mathrm{t}_{\mathrm{k}}+\mathrm{r}_{\mathrm{k}}\right) \mathrm{Nk} 3+ \\
\left.2 \dot{\mathrm{x}}_{\mathrm{k} 2}\left(\mathrm{t}_{\mathrm{k}}+\mathrm{r}_{\mathrm{k}}\right)(\mathrm{Nk} 4)\right]
\end{array} \\
& +\dot{\mathrm{x}}_{\mathrm{k} 1}\left(\mathrm{t}_{\mathrm{k}}\right)\left[\dot{\mathrm{x}}_{\mathrm{k} 2}\left(\mathrm{t}_{\mathrm{k}}\right) \mathrm{Nk} 5+\mathrm{x}_{\mathrm{k} 2}\left(\mathrm{t}_{\mathrm{k}}\right) \mathrm{Nk} 6+\dot{\mathrm{x}}_{\mathrm{k} 2}\right. \\
& \left.\left(t_{k}+r_{k}\right) N k 7+x_{k 2}\left(t_{k}+r_{k}\right) N k 8\right] \\
& +\mathrm{u}_{\mathrm{k} 1}\left(\mathrm{t}_{\mathrm{k}}\left[\mathrm{x}_{\mathrm{k} 2}\left(\mathrm{t}_{\mathrm{k}}\right) \mathrm{Nk} 9+\dot{\mathrm{x}}_{\mathrm{k} 2}\left(\mathrm{t}_{\mathrm{k}}\right) \mathrm{Nk} 10\right]\right. \\
& +\mathrm{h}_{\mathrm{k} 1}\left(\mathrm{t}_{\mathrm{k}}+\mathrm{r}_{\mathrm{k}}\right)\left[\mathrm{x}_{\mathrm{k} 2}\left(\mathrm{t}_{\mathrm{k}}+\mathrm{r}_{\mathrm{k}}\right) \mathrm{Nk} 11+\dot{\mathrm{x}}_{\mathrm{k} 2}\left(\mathrm{t}_{\mathrm{k}}+\mathrm{r}_{\mathrm{k}}\right) \mathrm{Nk} 2\right]
\end{aligned}
$$

Where:

$$
\begin{aligned}
& \mathrm{Nk} 1=\mathrm{a}_{\mathrm{k}}+\mathrm{ck} 2+\mathrm{pk}+\mathrm{wk} 2+\mathrm{nk}+\mathrm{wk} 1+2 \mathrm{vk} \\
& \mathrm{Nk} 2=\Delta_{\mathrm{k}} \mathrm{ck} 2+\Delta_{\mathrm{k}} \mathrm{pk}+\mathrm{wk} 1+\mathrm{vk} \\
& \mathrm{Nk} 3=\mathrm{ck}+2 \mathrm{ck} 1+\mathrm{ck} 2+\mathrm{pk}+\mathrm{mk} 1+\mathrm{mk} 2+\mathrm{nk} \\
& \mathrm{Nk} 4=\Delta_{\mathrm{k}}(\mathrm{ck}+\mathrm{ck} 1+\mathrm{ck} 2+\mathrm{mk} 2 \\
& \mathrm{Nk} 5=\Delta_{\mathrm{k}}^{2}(\mathrm{ck} 2+\mathrm{wk} 1) \\
& \mathrm{Nk} 6=\Delta_{\mathrm{k}}^{2}\left(\mathrm{ck} 2+\mathrm{mk} 2+\mathrm{v}_{\mathrm{k}}+\mathrm{wk} 1\right) \\
& \mathrm{Nk} 7=\Delta_{\mathrm{k}}^{2}(\mathrm{ck}+\mathrm{ck} 2) \\
& \mathrm{Nk} 8=\Delta_{\mathrm{k}}(\mathrm{ck}+\mathrm{ck} 1+\mathrm{ck} 2+\mathrm{pk}) \\
& \mathrm{Nk} 9=(\mathrm{mk}+\mathrm{wk}+\mathrm{qk}+\mathrm{dk}) \\
& \mathrm{Nk} 10=\Delta_{\mathrm{k}}(\mathrm{mk}+\mathrm{qk}) \\
& \mathrm{Nk} 11=\Delta_{\mathrm{k}}(\mathrm{ck}+\mathrm{ck} 1+\mathrm{ck} 2+\mathrm{pk}) \\
& \mathrm{Nk} 12=\Delta_{\mathrm{k}}^{2}(\mathrm{ck}+\mathrm{ck} 2)
\end{aligned}
$$


Now, we represent (14) by the following Eq. 15-18:

$\sum^{\mathrm{n}} \mathrm{x}_{\mathrm{k} 1}\left(\mathrm{t}_{\mathrm{k}}\right) \mathrm{V}_{11}+\dot{\mathrm{x}}_{\mathrm{k} 1}\left(\mathrm{t}_{\mathrm{k}}\right)$

$\sum_{\mathrm{k}=0} \dot{\mathrm{V}}_{11}+\mathrm{u}_{\mathrm{k} 1}\left(\mathrm{t}_{\mathrm{k}}\right) \mathrm{V}_{21}+\mathrm{h}_{\mathrm{k} 1}\left(\mathrm{t}_{\mathrm{k}}\right) \mathrm{V}_{31}$

Where:

$\mathrm{V}_{31}=\left[\mathrm{x}_{\mathrm{k} 2}\left(\mathrm{t}_{\mathrm{k}}-\mathrm{r}_{\mathrm{k}}\right) \mathrm{Nk} 11+\dot{\mathrm{x}}_{\mathrm{k} 2}\left(\mathrm{t}_{\mathrm{k}}+\mathrm{r}_{\mathrm{k}}\right) \mathrm{Nk} 12\right]$

$\mathrm{V}_{21}=\left[\mathrm{x}_{\mathrm{k} 2}\left(\mathrm{t}_{\mathrm{k}}\right) \mathrm{Nk} 9+\dot{\mathrm{x}}_{\mathrm{k} 2}\left(\mathrm{t}_{\mathrm{k}}\right) \mathrm{Nk} 10\right]$

To solve for $\mathrm{V}_{11}$, set:

$\Omega_{1}\left(\mathrm{t}_{\mathrm{k}}\right)=\mathrm{x}_{\mathrm{k} 2}\left(\mathrm{t}_{\mathrm{k}}\right) \mathrm{Nk} 1+\dot{\mathrm{x}}_{\mathrm{k} 2}\left(\mathrm{t}_{\mathrm{k}}\right) \mathrm{Nk} 2+2 \mathrm{x}_{\mathrm{k} 2}$

$\left(\mathrm{t}_{\mathrm{k}}+\mathrm{r}_{\mathrm{k}}\right) \mathrm{Nk} 3+2 \dot{\mathrm{x}}_{\mathrm{k} 2}\left(\mathrm{t}_{\mathrm{k}}+\mathrm{r}_{\mathrm{k}}\right) \mathrm{Nk} 4$

and:

$\mathrm{f}_{1}\left(\mathrm{t}_{\mathrm{k}}\right)=\dot{\mathrm{x}}_{\mathrm{k} 2}\left(\mathrm{t}_{\mathrm{k}}\right) \mathrm{Nk} 5+\mathrm{x}_{\mathrm{k} 2}\left(\mathrm{t}_{\mathrm{k}}\right) \mathrm{Nk} 6+\dot{\mathrm{x}}_{\mathrm{k} 2}$

$\left(\mathrm{t}_{\mathrm{k}}+\mathrm{r}_{\mathrm{k}}\right) \mathrm{Nk} 7+\mathrm{x}_{\mathrm{k} 2}\left(\mathrm{t}_{\mathrm{k}}+\mathrm{r}_{\mathrm{k}}\right) \mathrm{Nk} 8$

Now, both $\Omega_{1}\left(t_{k}\right)$ and $f_{1}\left(t_{k}\right)$ are continuous functions on $[0, T] . V_{11}\left(t_{k}\right)$ is continuous and at least twice differentiable on $[0, T]$. Hence $\Omega_{1}\left(t_{k}\right)-V_{11}\left(t_{k}\right)$ and $\mathrm{f}_{1}\left(\mathrm{t}_{\mathrm{k}}\right)-\mathrm{V}_{11}\left(\mathrm{t}_{\mathrm{k}}\right)$ are continuous on $[0, \mathrm{~T}]$. $\mathrm{x}(.) \in \mathrm{D}_{1}[0, \mathrm{~T}]$ such that $x(0)=x(T)$ and by (Polak, 1971), we have:

$\int_{0}^{T}\left\{\mathrm{x}_{\mathrm{k} 1}\left(\mathrm{t}_{\mathrm{k}}\right)\left[\Omega_{1}\left(\mathrm{t}_{\mathrm{k}}\right)-\mathrm{V}_{11}\right]+\dot{\mathrm{x}}_{\mathrm{k} 1}\left(\mathrm{t}_{\mathrm{k}}\right)\right.$
$\left.\left[\mathrm{f}_{1}\left(\mathrm{t}_{\mathrm{k}}\right)-\mathrm{V}_{11}\left(\mathrm{t}_{\mathrm{k}}\right)\right]\right\} \mathrm{dt}_{\mathrm{k}}=0$

Hence:

$\frac{\mathrm{d}}{\mathrm{dt}_{\mathrm{k}}}\left(\mathrm{f}_{1}\left(\mathrm{t}_{\mathrm{k}}\right)-\dot{\mathrm{V}}_{11}\left(\mathrm{t}_{\mathrm{k}}\right)=\Omega_{1}\left(\mathrm{t}_{\mathrm{k}}\right)-\mathrm{V}_{11}\left(\mathrm{t}_{\mathrm{k}}\right)\right)$

So:

$f_{1}\left(t_{k}\right)-\ddot{V}_{11}\left(t_{k}\right)=\Omega_{1}\left(t_{k}\right)-V_{11}\left(t_{k}\right), 0 \leq t_{k} \leq T$

Let:

$\ddot{\mathrm{V}}_{11}\left(\mathrm{t}_{\mathrm{k}}\right)-\mathrm{V}_{11}\left(\mathrm{t}_{\mathrm{k}}\right)=\dot{\mathrm{f}}_{1}\left(\mathrm{t}_{\mathrm{k}}\right)-\Omega_{1}\left(\mathrm{t}_{\mathrm{k}}\right)=\mathrm{q}\left(\mathrm{t}_{\mathrm{k}}\right)$

By:

$\dot{\mathrm{V}}_{11}\left(\mathrm{t}_{\mathrm{k}}\right)-\mathrm{V}_{11}\left(\mathrm{t}_{\mathrm{k}}\right)=\ddot{\mathrm{x}}_{\mathrm{k} 2}\left(\mathrm{t}_{\mathrm{k}}\right) \mathrm{Nk} 5+\dot{\mathrm{x}}_{\mathrm{k} 2}\left(\mathrm{t}_{\mathrm{k}}\right)$

$-\left[\mathrm{x}_{\mathrm{k} 2}\left(\mathrm{t}_{\mathrm{k}}\right) \mathrm{Nk} 1+\dot{\mathrm{x}}_{\mathrm{k} 2}\left(\mathrm{t}_{\mathrm{k}}\right) \mathrm{Nk} 2+2 \mathrm{x}_{\mathrm{k} 2}\left(\mathrm{t}_{\mathrm{k}}+\mathrm{r}_{\mathrm{ki}}\right)\right.$

$\left.\mathrm{Nk} 3+2 \dot{\mathrm{x}}_{\mathrm{k} 2}\left(\mathrm{t}_{\mathrm{k}}+\mathrm{r}_{\mathrm{k}}\right)(\mathrm{Nk} 4)\right]$
This is a second order ordinary differential equation that needs to be solved. So we impose the following initial conditions Eq. 19-23:

$\mathrm{V}_{11}(0)=\mathrm{p}_{1}$ and $\dot{\mathrm{V}}_{11}(0) \mathrm{r}_{1}$

where, $\mathrm{p}_{1}$ and $\gamma_{1}$ are to be determined. Let $\mathrm{Q}(\mathrm{s})=$ $\mathrm{L}\left(\mathrm{q}\left(\mathrm{t}_{\mathrm{k}}\right)\right)$ and $\mathrm{V}_{11}(\mathrm{~s})=\mathrm{L}\left(\mathrm{V}_{11}\left(\mathrm{t}_{\mathrm{k}}\right)\right)$ denote the Laplace transform of $\mathrm{q}\left(\mathrm{t}_{\mathrm{k}}\right)$ and $\mathrm{V}_{11}\left(\mathrm{t}_{\mathrm{k}}\right)$ respectively. Taking the Laplace transform of (22), we have Eq. 24:

$\mathrm{s}^{2} \mathrm{~V}_{11}(\mathrm{~s})-\mathrm{p}_{1} \mathrm{~s}-\gamma-\mathrm{V}_{11}(\mathrm{~s})=\mathrm{Q}(\mathrm{s})$

$\mathrm{V}_{11}(\mathrm{~s}) \frac{\mathrm{Q}(\mathrm{s})}{\mathrm{s}^{2-1}}+\frac{\mathrm{p}_{1} \mathrm{~s}}{\mathrm{~s}^{2-1}}+\frac{\gamma_{1}}{\mathrm{~s}^{2-1}}$

We take the inverse Laplace transform of (25) and using convolution theorem for the first term on the right to obtain Eq. 26 and 27:

$$
\begin{aligned}
& V_{11}\left(t_{k}\right)=\int_{0}^{T} d_{k}\left(s_{k}\right) \sinh \left(t_{k}-s_{k}\right) \\
& \cosh \left(t_{k}\right)+\sinh \left(t_{k}\right) \\
&=-\sinh (T) f_{1}(0)+\int_{0}^{T} f_{1}\left(s_{k}\right) \cosh \left(t_{k}-s_{k}\right) d s_{k} \\
& \Omega_{1}\left(t_{k}\right) \sinh \left(t_{k}-s_{k}\right) d s_{k}
\end{aligned}
$$

$+\mathrm{p}_{1} \cosh \left(\mathrm{t}_{\mathrm{k}}\right)+\gamma_{1} \sinh \left(\mathrm{t}_{\mathrm{k}}\right)$

where, $\mathrm{qs}_{\mathrm{k}}==\mathrm{f}_{1}\left(\mathrm{~s}_{\mathrm{k}}-\Omega_{1}\left(\mathrm{~s}_{\mathrm{k}}\right)\right)$ and Eq. 28:

$\tau_{1} \frac{1}{\sinh (\mathrm{T})}\left\{\begin{array}{r}\left\{\mathrm{f}_{1}\left(\mathrm{~s}_{\mathrm{k}}\right) \cosh \left(\mathrm{T}-\mathrm{s}_{\mathrm{k}}\right) \mathrm{ds}_{\mathrm{k}}+\right. \\ \sinh (\mathrm{T}) \mathrm{f}_{1}(0)-\int_{0}^{\mathrm{T}} \int_{0}^{\mathrm{T}} \Omega\left(\mathrm{s}_{\mathrm{k}}\right) \sinh \left(\mathrm{T}-\mathrm{s}_{\mathrm{k}}\right) \mathrm{ds}_{\mathrm{k}}- \\ \Omega(0) \cosh (\mathrm{T})+\Omega_{1}(\mathrm{~T})\end{array}\right\}$

Now, solve for $\mathrm{u}_{\mathrm{k} 2}$ by setting $\mathrm{x}_{\mathrm{k} 2}\left(\mathrm{t}_{\mathrm{k}}\right)=\mathrm{h}_{\mathrm{k} 2}\left(\mathrm{t}_{\mathrm{k}}\right)=$ $0 \rightarrow \mathrm{x}_{\mathrm{k} 2}\left(\mathrm{t}_{\mathrm{k}}\right)=0$ in EQ. 5.2, following the same sequence as from the beginning of the proof to equation(28), we then have Eq. 29-34:

$\mathrm{V}_{32}\left(\mathrm{t}_{\mathrm{k}}\right)=\mathrm{u}_{\mathrm{k} 2}\left(\mathrm{t}_{\mathrm{k}}\right) \Delta_{\mathrm{k}} \mathrm{mk}$

$\mathrm{V}_{22}\left(\mathrm{t}_{\mathrm{k}}\right)=\mathrm{u}_{\mathrm{k} 2}\left(\mathrm{t}_{\mathrm{k}}\right) \mathrm{bk}$

$\mathrm{f}_{2}\left(\mathrm{t}_{\mathrm{k}}\right)=\mathrm{u}_{\mathrm{k} 2}\left(\mathrm{t}_{\mathrm{k}}\right) \Delta_{\mathrm{k}} \mathrm{qk}$

$\Omega_{2}\left(\mathrm{t}_{\mathrm{k}}\right)=\mathrm{u}_{\mathrm{k} 2}\left(\mathrm{t}_{\mathrm{k}}\right)\left(\mathrm{q}_{\mathrm{k}}+\mathrm{z}_{\mathrm{k}}\right)+$

$\mathrm{u}_{\mathrm{k} 2}\left(\mathrm{t}_{\mathrm{k}}+\gamma_{\mathrm{k}}\right)\left(2 \mathrm{mk}+2 \mathrm{wk}+\Delta_{\mathrm{k}} \mathrm{mk}\right)$ 


$$
\begin{aligned}
& \mathrm{V}_{12}\left(\mathrm{t}_{\mathrm{k}}\right)=-\sinh (\mathrm{T}) \mathrm{f}_{2}(0)+\int_{0}^{\mathrm{T}} \mathrm{f}_{2}\left(\mathrm{~s}_{\mathrm{k}}\right) \cosh \left(\mathrm{t}_{\mathrm{k}}-\mathrm{s}_{\mathrm{k}}\right) \mathrm{ds}_{\mathrm{k}} \\
& -\int_{0}^{\mathrm{T}} \Omega_{2}\left(\mathrm{~s}_{\mathrm{k}}\right) \sinh \left(\mathrm{t}_{\mathrm{k}}-\mathrm{s}_{\mathrm{k}}\right) \mathrm{ds}_{\mathrm{k}}+\mathrm{p}_{2} \cosh \left(\mathrm{t}_{\mathrm{k}}\right)+\mathrm{r}_{2} \sinh \left(\mathrm{t}_{\mathrm{k}}\right) \\
& \tau_{2}=\frac{1}{\sinh (\mathrm{T})}\left\{\sinh (\mathrm{T}) \mathrm{f}_{2}(0)-\int_{0}^{\mathrm{T}} \mathrm{f}_{2}\left(\mathrm{~s}_{\mathrm{k}}\right) \cosh \left(\mathrm{T}-\mathrm{s}_{\mathrm{k}}\right)\right. \\
& \mathrm{ds}_{\mathrm{k}}+\int_{0}^{\mathrm{T}} \Omega_{2}\left(\mathrm{~s}_{\mathrm{k}}\right) \sinh \left(\mathrm{T}-\mathrm{s}_{\mathrm{k}}\right) \mathrm{ds}_{\mathrm{k}}-\Omega_{1} \\
& \left.(0) \cosh (\mathrm{T})+\Omega_{1}(\mathrm{~T})\right\}
\end{aligned}
$$

Finally, we solve for $\mathrm{h}_{\mathrm{k} 2}\left(\mathrm{t}_{\mathrm{k}}\right)$ by setting $\mathrm{x}_{\mathrm{k} 2}\left(\mathrm{t}_{\mathrm{k}}\right)=$ $\mathrm{u}_{\mathrm{k} 2}\left(\mathrm{t}_{\mathrm{k}}\right)=0 \rightarrow \mathrm{x}_{\mathrm{k} 2}\left(\mathrm{t}_{\mathrm{k}}\right)=0$, in Eq. 10 and following the same sequence as from the beginning of the proof to (28), we have Eq. 35-41:

$$
\begin{aligned}
& \mathrm{V}_{22}\left(\mathrm{t}_{\mathrm{k}}\right)=\mathrm{h}_{\mathrm{k} 2}\left(\mathrm{t}_{\mathrm{k}}\right)\left[\Delta_{\mathrm{k}}\left(\Delta_{\mathrm{k}}+\mathrm{c}_{\mathrm{k}}+\mathrm{c}_{\mathrm{k} 1}\right)\right] \\
& \left.\mathrm{V}_{23}\left(\mathrm{t}_{\mathrm{k}}\right)=\mathrm{h}_{\mathrm{k} 2}\left(\mathrm{t}_{\mathrm{k}}\right)\left[\Delta_{\mathrm{k}} \mathrm{m}_{\mathrm{k}}\right)\right] \\
& \mathrm{f}_{3}\left(\mathrm{~T}_{\mathrm{k}}\right)=\mathrm{h}_{\mathrm{k} 2}\left(\mathrm{t}_{\mathrm{k}}\right)\left(\Delta_{\mathrm{k}}^{2}\right)
\end{aligned}
$$

$$
\Omega_{3}\left(\mathrm{t}_{\mathrm{k}}\right)=\mathrm{h}_{\mathrm{k} 2}\left(\mathrm{t}_{\mathrm{k}}\right)\left[\Delta_{\mathrm{k}}\left(\mathrm{c}_{\mathrm{k} 2}+\mathrm{p}_{\mathrm{k}}+\mathrm{c}_{\mathrm{k}} \Delta_{\mathrm{k}}+\mathrm{c}_{\mathrm{k}}+\mathrm{c}_{\mathrm{k} 1}\right)\right]
$$

$$
\begin{array}{r}
V_{13}\left(t_{k}\right)=-\sinh (T) f_{3}(0)+\int_{0}^{T} f_{3}\left(s_{k}\right) \cosh \left(t_{k}-s_{k}\right) d s_{k}- \\
\int_{0}^{T} \Omega_{3}\left(t_{k}\right) \sinh \left(t_{k}-s_{k}\right) d s_{k}+ \\
p_{3} \cosh \left(t_{k}\right)+\gamma_{3} \sinh \left(t_{k}\right)
\end{array}
$$

$$
\begin{aligned}
& \tau_{3}= \frac{1}{\sinh (\mathrm{T})}\left\{\sinh (\mathrm{T}) \mathrm{f}_{3}(0)-\int_{0}^{\mathrm{T}} \mathrm{f}_{3}\left(\mathrm{~s}_{\mathrm{k}}\right) \cosh \left(\mathrm{T}-\mathrm{s}_{\mathrm{k}}\right)\right. \\
& \mathrm{ds}_{\mathrm{k}}+\int_{0}^{\mathrm{T}} \Omega_{3}\left(\mathrm{~s}_{\mathrm{k}}\right) \sinh \left(\mathrm{T}-\mathrm{s}_{\mathrm{k}}\right) \mathrm{ds}_{\mathrm{k}}- \\
&\left.\Omega_{3}(0) \cosh (\mathrm{T})+\Omega_{3}(\mathrm{~T})\right\}
\end{aligned}
$$

Having constructed operator $\mathrm{V}$, written as:

$$
\mathrm{V}=\left(\begin{array}{lll}
\mathrm{V}_{11} & \mathrm{~V}_{12} & \mathrm{~V}_{13} \\
\mathrm{~V}_{21} & \mathrm{~V}_{22} & \mathrm{~V}_{23} \\
\mathrm{~V}_{31} & \mathrm{~V}_{32} & \mathrm{~V}_{33}
\end{array}\right)
$$

with the generalized scheme and associated operator, a program is written using the Conjugate Gradient Method Algorithm (Polak, 1971) to execute and examine the convergence profile and the geometric ratio profile.Next we give the theoretical backing for the geometric convergence ratio.
Convergence analysis of the discretized scheme: This section shows that the discretized penalty extended conjugate gradient method converges geometrically by(Shannon, 1978; Aruchunan and Sulaiman, 2010).

Definition 6.1 The sequence $\left\{z_{n}\right\}$ generated by the discretized conjugate gradient method of $\mathrm{K}$ converges geometrically to $z^{\bullet}$ such that:

$$
\lim _{n \rightarrow \infty} \frac{\left|z_{n+1}-z^{\bullet}\right|}{\left|z_{n}-z^{*}\right|}=p<1
$$

where, $p$ is the convergence ratio.

Now we sate the conjugate gradient method algorithm using our developed scheme for quadratic objective function:

$f(x)=\frac{1}{2} x^{T} A x+b x+c$

Step 1: Pick the initial solution $\mathrm{z}_{0}\left(\mathrm{t}_{\mathrm{k}}\right)$ arbitrarily, where $\mathrm{z}_{0}\left(\mathrm{t}_{\mathrm{k}}\right)=\left(\mathrm{x}_{0}, \mathrm{u}_{\mathrm{o}}, \mathrm{h}_{0}\right) \in \mathrm{K}$

Step 2: Compute the initial direction; $\mathrm{p}_{0}=-(\mathrm{Ax}+\mathrm{a})=$ $\mathrm{g}_{0}$

Step 3: Update the initial guess as follows:

$$
\begin{aligned}
& \mathrm{x}_{\mathrm{k}+1}\left(\mathrm{t}_{\mathrm{k}}\right)=\mathrm{x}_{\mathrm{k}}\left(\mathrm{t}_{\mathrm{k}}\right)+\mathrm{a}_{\mathrm{x}} \mathrm{p}_{\mathrm{x}, \mathrm{k}} \\
& \mathrm{u}_{\mathrm{k}+1}\left(\mathrm{t}_{\mathrm{k}}\right)=\mathrm{u}_{\mathrm{k}}\left(\mathrm{t}_{\mathrm{k}}\right)+\mathrm{a}_{\mathrm{k}} \mathrm{p}_{\mathrm{u}, \mathrm{k}} \\
& \mathrm{h}_{\mathrm{k}+1}\left(\mathrm{t}_{\mathrm{k}}\right)=\mathrm{h}_{\mathrm{k}}\left(\mathrm{t}_{\mathrm{k}}\right)+\mathrm{a}_{\mathrm{k}} \mathrm{p}_{\mathrm{h}, \mathrm{k}} \\
& \text { where, } \mathrm{a}_{\mathrm{k}}=\frac{\left(\mathrm{g}_{\mathrm{k}}, \mathrm{g}_{\mathrm{k}}\right)}{\mathrm{p}_{\mathrm{k}}, \mathrm{ap}_{\mathrm{k}}}
\end{aligned}
$$

Step 4: Update the gradient and stepsize at the $(\mathrm{k}+1)$ th step as follows:

$$
\begin{aligned}
& g_{x, k+1}=g_{x, k}+a_{k} A p_{x, k} \\
& g_{u, k+1}=g_{u, k}+a_{k} A p_{u, k} \\
& g_{h, k+1}=g_{h, k}+a_{k} A p_{h, k} \quad \text { where } g=\left(\begin{array}{l}
g_{x, k} \\
g_{u, k} \\
g_{h, k}
\end{array}\right) \text { and } p=\left(\begin{array}{l}
p_{x, k} \\
p_{u, k} \\
p_{h, k}
\end{array}\right)
\end{aligned}
$$

and:

$$
\begin{aligned}
& P_{x, k+1}=-g_{x, k+1}+\beta_{k} p_{x, k} \\
& P_{u, k+1}=-g_{u, k+1}+\beta_{k} p_{u, k} \\
& P_{h, k+1}=-g_{x, k+1}+\beta_{k} p_{h, k} \text { where } g=\frac{\left(g_{k+1}, g_{k+1}\right)}{\left(g_{k}, g_{k}\right)} \\
& \text { Setting Eq. } 11 \text { equal to Eq. 42-45: } \\
& \begin{aligned}
V z_{k 2}\left(t_{k}\right)=\left(\begin{array}{l}
V_{11} z_{x}\left(t_{k}\right)+V_{12} z_{x}\left(t_{k}\right)+V_{13} z_{x}\left(t_{k}\right)+ \\
V_{21} z_{x}\left(t_{k}\right)+V_{22} z_{x}\left(t_{k}\right)+V_{23} z_{x}\left(t_{k}\right)+ \\
V_{31} z_{x}\left(t_{k}\right)+V_{32} z_{x}\left(t_{k}\right)+V_{33} z_{x}\left(t_{k}\right)+
\end{array}\right) \\
=\left(\begin{array}{l}
\bar{V} z_{1}\left(t_{k}\right) \\
\bar{V} z_{2}\left(t_{k}\right) \\
\bar{V} z_{3}\left(t_{k}\right)
\end{array}\right)
\end{aligned}
\end{aligned}
$$


Where:

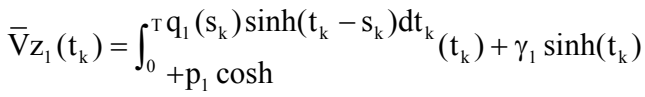

$$
\begin{aligned}
& +\int_{0}^{\mathrm{T}} \mathrm{q}_{2}\left(\mathrm{~s}_{\mathrm{k}}\right) \sinh \left(\mathrm{t}_{\mathrm{k}}-\mathrm{s}_{\mathrm{k}}\right) \mathrm{dt}_{\mathrm{k}}+{ }_{\left(\mathrm{t}_{\mathrm{k}}\right)+\gamma_{2} \sinh \left(\mathrm{t}_{\mathrm{k}}\right)+} \\
& \int_{0}^{\mathrm{T}} \mathrm{q}_{3}\left(\mathrm{~s}_{\mathrm{k}}\right) \sinh \left(\mathrm{t}_{\mathrm{k}}-\mathrm{s}_{\mathrm{k}}\right) \mathrm{dt}_{\mathrm{k}}\left(\mathrm{t}_{\mathrm{k}}\right)+\gamma_{3} \sinh \left(\mathrm{t}_{\mathrm{k}}\right) \\
& \overline{\mathrm{V}} \mathrm{z}_{1}\left(\mathrm{t}_{\mathrm{k}}\right)=\mathrm{X}_{\mathrm{K} 2}\left(\mathrm{t}_{\mathrm{k}}\right)\left(\mathrm{m}_{\mathrm{k}}+\mathrm{w}_{\mathrm{k}}+\mathrm{q}_{\mathrm{k}}+\mathrm{d}_{\mathrm{k}}\right)+\dot{\mathrm{x}}_{\mathrm{k} 2}\left(\mathrm{t}_{\mathrm{k}}\right)\left(\Delta_{\mathrm{k}}\right) \\
& \left(\mathrm{m}_{\mathrm{k}}+\mathrm{q}_{\mathrm{k}}\right)+\mathrm{u}_{\mathrm{k} 2}\left(\mathrm{t}_{\mathrm{k}}\right) \mathrm{b}_{\mathrm{k}}+\mathrm{h}_{\mathrm{k} 2}\left(\mathrm{t}_{\mathrm{k}}\right)\left(\Delta_{\mathrm{k}} \mathrm{m}_{\mathrm{k}}\right) \\
& \overline{\mathrm{V}} \mathrm{z}_{3}\left(\mathrm{t}_{\mathrm{k}}\right)=\mathrm{X}_{\mathrm{K} 2}\left(\mathrm{t}_{\mathrm{k}}+\gamma_{\mathrm{k}}\right)\left(\left(\Delta_{\mathrm{k}}\right)\left(\mathrm{c}_{\mathrm{k}}+\mathrm{c}_{\mathrm{k} 1}+\mathrm{c}_{\mathrm{k} 2}+\mathrm{p}_{\mathrm{k}}\right)\right. \\
& +\dot{\mathrm{x}}_{\mathrm{k} 2}\left(\mathrm{t}_{\mathrm{k}}\right)\left(\Delta_{\mathrm{k}}^{2}\left(\mathrm{c}_{\mathrm{k}}+2 \mathrm{c}_{\mathrm{k} 2}\right)+\mathrm{u}_{\mathrm{k} 2}\left(\mathrm{t}_{\mathrm{k}}\right) \mathrm{b}_{\mathrm{k}}+\mathrm{h}_{\mathrm{k} 2}\right) \\
& \left(\mathrm{t}_{\mathrm{k}}\right)\left(\Delta_{\mathrm{k}}\left(\mathrm{m}_{\mathrm{k}}+\mathrm{c}_{\mathrm{k}}+\mathrm{c}_{\mathrm{k} 1}\right)\right)
\end{aligned}
$$

Now, we state and prove the geometric convergence property of the developed scheme.

Theorem 6.1: The sequence of estimated solutions $\left\{z_{\mathrm{k}}\right\}$ generated by the discretized penalty method converges to $\mathrm{z}^{*}$ with ratio $\omega$ given by:

$$
\omega^{2}=1-\frac{\tau}{\left|\mathrm{z}_{0}\right|}
$$

where, $\tau=\max \left\{\frac{\left\|V z_{n}\right\|^{3}}{V p_{n}, p_{n}}\right\}$ and $z_{0}$ is the initial guess.

Proof: Define:

$\mathrm{f}(\mathrm{z})=\left(\mathrm{z}-\mathrm{z}^{*}, \mathrm{Vz}-\mathrm{z}^{*}\right) \mathrm{k}$ and $\mathrm{z}^{\mathrm{t}}\left(\mathrm{t}_{\mathrm{k}}\right)=\left(\mathrm{x}_{\mathrm{k}}, \mathrm{u}_{\mathrm{k}}, \mathrm{h}_{\mathrm{k}}\right)$

With optimality condition $\mathrm{Vz}^{*}=0$. From (46)

$\mathrm{f}\left(\mathrm{z}_{\mathrm{k}}\right)=\left(\mathrm{Z}_{\mathrm{k}}-\mathrm{Z}^{*}, \mathrm{VZ}_{\mathrm{k}}-\mathrm{Z}^{*}\right)_{\mathrm{k}}$

$=\left(\mathrm{z}_{\mathrm{k}}, \mathrm{Vz_{ \textrm {k } }}\right)-\left(\mathrm{z}_{\mathrm{k}}^{*}, \mathrm{Vz}_{\mathrm{k}}\right)_{\mathrm{k}}$

$\mathrm{f}\left(\mathrm{z}_{\mathrm{k}+1}\right)=\left(\mathrm{z}_{\mathrm{k}}+\mathrm{a}_{\mathrm{k}} \mathrm{p}_{\mathrm{k}}-\mathrm{z}^{*}, \mathrm{~V}\left(\mathrm{z}_{\mathrm{n}}+\mathrm{a}_{\mathrm{k}} \mathrm{p}_{\mathrm{k}}-\mathrm{z}^{*}, \mathrm{~V}\right)\right)_{\mathrm{k}}$

$=\left(z_{k}, V z_{k}\right)_{k}+a_{k}\left(z_{k}, V p_{k}\right)_{k}+a_{k}\left(p_{k}, V z_{k}\right)_{k}+a_{k}\left(p_{k}, V p_{k}\right)_{k}$

$-\left(\mathrm{z}^{*}, \mathrm{VZ}_{\mathrm{k}}\right)_{\mathrm{k}}-\mathrm{a}_{\mathrm{k}}\left(\mathrm{z}^{*}, \mathrm{Vp}_{\mathrm{k}}\right)_{\mathrm{k}}$

Therefore:

$f\left(z_{k}\right)-f\left(z_{k+1}\right)=\frac{\left(V z_{k}, V z_{k}\right)_{k}}{\left(p_{k}, V p_{k}\right)_{k}} \cdot \frac{f\left(z_{k}\right)}{\left(V z_{k}, z_{k}\right)_{k}}$

since $\mathrm{V}$ is self-adjoint and $\mathrm{Vz} *=0$.Again Eq. 46-50:

$$
\left(\mathrm{Vz}_{\mathrm{k}}, \mathrm{z}_{\mathrm{k}}\right)_{\mathrm{k}}\left(\mathrm{Vz}_{\mathrm{k}}, \mathrm{z}_{0}\right)+\sum_{\mathrm{j}=0}^{\mathrm{N}-1}\left(\mathrm{Vz}_{\mathrm{k}}, \mathrm{p}_{\mathrm{j}}\right)
$$

and:

$$
\sum_{\mathrm{j}=0}^{\mathrm{N}-1}\left(\mathrm{Vz}_{\mathrm{k}}, \mathrm{p}_{\mathrm{j}}\right)_{\mathrm{k}}=0, \mathrm{k} \neq \mathrm{j}
$$

Hence:

$\left(\mathrm{VZ}_{\mathrm{k}}, \mathrm{Z}_{\mathrm{k}}\right)_{\mathrm{k}}=\left(\mathrm{Vz}_{\mathrm{k}}, \mathrm{Z}_{0}\right)$

$\mathrm{V}$ is a bounded operator then there are positive numbers $\mathrm{m}$ and $\mathrm{M}$ such that for every $\mathrm{z}$ in $\mathrm{K}$ :

$\mathrm{m}\left\|\mathrm{z}-\mathrm{z}^{\bullet}\right\|^{2} \leq\left\|\mathrm{V}\left(\mathrm{z}-\mathrm{z}^{\bullet}\right)\right\|^{2} \leq \mathrm{M}\left\|\mathrm{z}-\mathrm{z}^{\bullet}\right\|^{2}$

Hence:

$\left(\mathrm{Vz}_{\mathrm{k}}, \mathrm{z}_{\mathrm{k}}\right) \leq\left\|\mathrm{Vz_{ \textrm {n } }}\right\|\left\|\mathrm{z}_{0}\right\|$

Substitute inequality (50) in Eq. 47 to obtain:

$f\left(z_{k}\right)-f\left(z_{k+1}\right) \geq \frac{\left(V z_{k}, V z_{k}\right)_{k}^{2} f\left(z_{k}\right)}{\left(p_{k}, V p_{k}\right)_{k}\left\|V z_{k}\right\|\left\|z_{0}\right\|}$

Using (6.10):

$f\left(z_{k}\right) \geq m\left\|z_{k}-z^{\bullet}\right\|$

and Eq. 51 becomes Eq. 52 and 53:

$\frac{\left\|z_{k+1}-z^{\bullet}\right\|}{\left\|z_{k}-z^{\bullet}\right\|} \leq \frac{f\left(z_{k+1}\right)}{f\left(z_{k}\right)} \leq 1-\frac{\left\|V z_{k}\right\|^{3}}{\left(p_{k}, V p_{k}\right)_{k}\left\|z_{0}\right\|}$

Therefore:

$$
\begin{aligned}
& \frac{\left\|z_{k+1}-z^{\bullet}\right\|}{\left\|z_{k}-z^{\bullet}\right\|} \leq 1-\frac{\left\|V z_{k}\right\|^{3}}{\left(p_{k}, V p_{k}\right)_{k}\left\|z_{0}\right\|}, \\
& \text { where } \tau=\sup \left\{\frac{\left\|V z_{k}\right\|^{3}}{\left(p_{k}, V p_{k}\right)_{k}}\right\}
\end{aligned}
$$

This completes the proof.

Numerical example: This example validates Theorem (9) and Theorem(39) by minimizing the following quadratic functional with real coefficients Eq. 54 and 55:

$\operatorname{Minimize~} L(t, x, u)_{k}=\int_{0}^{T}\left(x^{2}(t)+u^{2}(t)\right) d t$

subject to the linear restoring dynamic equation: 


\section{J. Math. \& Stat., 7 (2): 116-123, 2011}

$$
\dot{\mathrm{x}}(\mathrm{t}-0.2)+\dot{\mathrm{x}}(\mathrm{t})=2 \mathrm{x}(\mathrm{t})+5 \mathrm{u}(\mathrm{t})
$$$$
\mathrm{x}(0)=\dot{\mathrm{x}}(\mathrm{t})=\mathrm{h}(\mathrm{t}), \mathrm{t} \in[-0.2,0]
$$

For better understanding of the results, the following abbreviations and notations shall be invoked:

$\mathrm{J}\left(\mathrm{x}_{\mathrm{k}}, \mathrm{x}_{\mathrm{k}}(\mathrm{t}-\gamma), \mathrm{u}_{\mathrm{k}}\right)=$ the objective functional

$\mathrm{F}\left(\mathrm{x}_{\mathrm{k}}, \mathrm{x}_{\mathrm{k}}(\mathrm{t}-\gamma), \mathrm{u}_{\mathrm{k}}, \mu\right)=$ the penalized functional

The analytic solution is given as 0.2328 by (Olotu and Adekunle, 2010).

\section{RESULTS AND DISCUSSION}

For every fixed penalty constant per cycle, it is seen that convergence is attained as evidenced by the values of the objective functional. For instance, for penalty constant $\mu=0.01$, the objective functional values initiating at 0.26 and ending at .2537889 , a value attained at the sixth iteration compares more favorably with the analytic solution 0.2328 . The same applies to every cycle with at most six iterations. As the penalty parameter increases, the better the approximation to the analytic solution. Also, the geometric convergence ratio profile is attained favorably, since the geometric ratio is greater than zero and less than one, as exhibited under the geometric ratio column in Table 1. For instance, for $\mu=0.01$, we see that the geometric convergence ratios; $0.785294,0.984049,0.998686,0.999888$ and 0.999999 and are all greater zero and less than one. The same information is seen for other cycles.

Table 1: Numerical results comparable to the analytical solution (0.2328)

\begin{tabular}{|c|c|c|c|c|c|}
\hline Parameters & Iteration No & Obj. functional & $\begin{array}{l}\text { Constr } \\
\text { satisfaction }\end{array}$ & $\begin{array}{l}\text { Penalized } \\
\text { functional }\end{array}$ & Geometric ratio \\
\hline \multirow[t]{6}{*}{$\mu=0.01$} & 1 & 0.2600000 & 7.290000 & 0.3329000 & \\
\hline & 2 & 0.2541600 & 7.062863 & 0.3248046 & 0.7852940 \\
\hline & 3 & 0.2538193 & 7.012422 & 0.3239435 & 0.9840490 \\
\hline & 4 & 0.2537917 & 7.006729 & 0.3238590 & 0.9986860 \\
\hline & 5 & 0.2537892 & 7.006135 & 0.3238590 & 0.9998880 \\
\hline & 6 & 0.2537889 & 7.006078 & 0.3238497 & 0.9999990 \\
\hline \multirow[t]{6}{*}{$\mu=0.002$} & 1 & 0.2600000 & 7.290000 & 0.2745800 & \\
\hline & 2 & 0.2544980 & 7.060028 & 0.3686180 & 0.7977200 \\
\hline & 3 & 0.2541324 & 7.011543 & 0.2686180 & 0.9833380 \\
\hline & 4 & 0.2541083 & 7.006103 & 0.2681205 & 0.9986360 \\
\hline & 5 & 0.2540550 & 7.005534 & 0.2681166 & 0.9974980 \\
\hline & 6 & 0.2540530 & 7.005477 & 0.2681162 & 0.9999250 \\
\hline \multirow[t]{6}{*}{$\mu=0.003$} & 1 & 0.2600000 & 7.290000 & 0.2818700 & \\
\hline & 2 & 0.2546610 & 7.060249 & 0.2756469 & 0.8037130 \\
\hline & 3 & 0.2541061 & 7.011531 & 0.2756469 & 0.8037130 \\
\hline & 4 & 0.2540772 & 7.006063 & 0.2750954 & 0.9986435 \\
\hline & 5 & 0.2540745 & 7.005490 & 0.2750910 & 0.9998730 \\
\hline & 6 & 0.2540742 & 7.005434 & 0.2750905 & 0.9999800 \\
\hline \multirow[t]{6}{*}{$\mu=0.04$} & 1 & 0.2600000 & 7.290000 & 0.2891600 & \\
\hline & 2 & 0.2544343 & 7.060483 & 0.2826762 & 0.7953780 \\
\hline & 3 & 0.2540749 & 7.011533 & 0.2821210 & 0.9833870 \\
\hline & 4 & 0.2540462 & 4.006033 & 0.2820703 & 0.9986500 \\
\hline & 5 & 0.2540435 & 7.005458 & 0.2820653 & 0.9998720 \\
\hline & 6 & 0.2540432 & 7.005402 & 0.2820648 & 0.9999850 \\
\hline \multirow[t]{6}{*}{$\mu=0.05$} & 1 & 0.2600000 & 7.290000 & 0.2964500 & \\
\hline & 2 & 0.2544024 & 7.060731 & 0.2897016 & 0.7942050 \\
\hline & 3 & 0.2540436 & 7.011546 & 0.2891040 & 0.9833900 \\
\hline & 4 & 0.2540151 & 7.006015 & 0.2890452 & 0.9986580 \\
\hline & 5 & 0.2540124 & 7.005437 & 0.2890396 & 0.9998720 \\
\hline & 6 & 0.2540122 & 7.00538 & 0.2890391 & 0.9999900 \\
\hline
\end{tabular}




\section{CONCLUSION}

Considering the minimum of the objective functional values for each cycle, it is readily seen that the numerical solution approximates the analytic solution 0.2328 within an error tolerance of 0.021 . The convergence analysis is equally favorable. This shows that the developed scheme for the solution of optimal problem constrained by evolution equation is adequately effective and efficient.

Recommendation: The results obtained and the information gathered from the geometric ratio profile imply that a solution of an hypothetical constrained problem can be approximated with this scheme, even without a prior knowledge of the analytical solution.

\section{REFERENCES}

Aruchunan, E. and J. Sulaiman, 2010. Numerical solution of second-order linear fredholm integrodifferential equation using generalized minimal residual method. Am. J. Applied Sci., 7: 780-783. DOI: 10.3844/ajassp.2010.780.783

Di Pillo, G. and L. Grippo, 1979. A new class of augmented lagrangians in nonlinear programming. SIAM, J. Cont. Optimiz., 17: 618-628.
Fletcher, R. and C.M. Reeves, 1964. Function Minimization by Conjugate Gradients. Comput. J., 7: 149-154. DOI: $10.1093 /$ comjn1/7.2.149

Glad, S.T., 1979. A combination of penalty function and multiplier methods for solving optimal control problems. J. Optimiz. Theory Appl., 28: 303-329. DOI: $10.1007 / \mathrm{BF} 00933377$

Hestenes, M.R., 1969. Multiplier and gradient methods. J. Optimiz. Theory Appl., 4: 303-320. DOI: 10.1007/BF00927673

Ibiejugba, M.A. and P. Onumanyi, 1984. A control operator and some of its applications. J. Math. Anal. Appl., 103: 31-47. DOI: 10.1016/0022247X(84)90154-9

Olotu, O. and A.I. Adekunle, 2010. Analytic and numeric solutions of discretized constrained optimal control problem with vector and matrix coefficients. Int. J. Adv. Modell. Optimiz., 12: 119-131.

Polak, E., 1971. Computational Methods in Optimization: A Unified Approach. 1st Edn., Academic press, London, ISBN-10: 0125593503, pp: 329.

Shannon, D.F., 1978. On the convergence of a new conjugate gradient algorithm. SIAM, J. Numerical Anal., 15: 1247-1257. 